\title{
DÉCEMBRE 1970
}

52. ANNÉE - N• 624

\section{revue internationale de la croix-rouge}

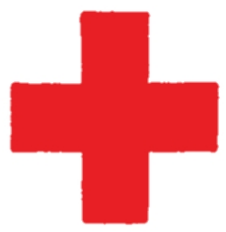

INTER ARMA CARITAS

GENËVE

COMITÉ INTERNATIONAL DE LA CROIX-ROUGE FONDE EN 1863 


\title{
COMITÉ INTERNATIONAL DE LA CROIX-ROUGE
}

MM. MARCEL A. NAVILLE, president (membre depuis 1967)

HANS BACHMANN, docteur en droit, Stadtrat de Winterthur, vice-président (1958)

JACQUES FREYMOND, docteur ès lettres, directeur de l'Institut universitaire de hautes études internationales, professeur à l'Université de Genève, vice-président (1959)

MARTIN BODMER, docteur en philosophie h.c. (1940)

PAUL RUEGGER, ambassadeur, président du CICR de 1948 à 1955 (1948)

RODOLFO OLGIATI, docteur en médecine h.c., directeur du Don Suisse de 1944 à 1948 (1949

GUILLAUME BORDIER, ingénieur dipl. E.P.F., M.B.A. Haryard, banquier (1955)

DIETRICH SCHINDLER, docteur en droit, professeur à l'Université de Zurich (1961)

HANS MEULI, docteur en médecine, colonel brigadier, chef du Service de santé de l'armée suisse de 1946 à 1960 (1961)

MUe MARJORIE DUVILLARD, infirmière (1961)

MM. MAX PETITPIERRE, docteur en droit, ancien président de la Confédération suisse (1961)

ADOLPHE GRAEDEL, député au Conseil national suisse de 1951 à 1963, ancien secrétaire général de la Fédération internationale des ouvriers sur métaux (1965)

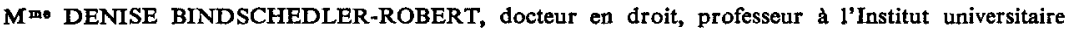
de hautes études internationales (1967)

MM. JACQUES F. DE ROUGEMONT, docteur en médecine (1967)

ROGER GALLOPIN, docteur en droit, ancien directeur général (1967)

JEAN PICTET, docteur en droit, president de la Commission juridique (1967)

WALDEMAR JUCKER, docteur en droit, secrétaire de l'Union syndicale suisse (1967)

HARALD HUBER, docteur en droit, juge fédéral (1969)

VICTOR H. UMBRICHT, docteur en droit, administrateur (1970)

\begin{abstract}
Membres honoraires: M. JACQUES CHENEVIERE, vice-président d'honneur; M 10 LUCIE ODIER, vice-présidente d'honneur; MM. CARL J. BURCKHARDT, PAUL CARRY, $M^{\text {me }}$ MARGUERITE GAUTIER-VAN BERCHEM, MM. SAMUEL A. GONARD, EDOUARD DE HALlER, PAUL LOGOZ, FREDÉrIC SIORDET, ALFREDO VANNOTTI, ADOLF VISCHER.
\end{abstract}

Direction: MM. JEAN-LOUIS LE FORT, secrétaire génêral;

RAYMOND COURVOISIER, assistant spécial du président et directeur du département des opérations;

ClAUDe PILloUd, directeur du département des principes et du droit. 


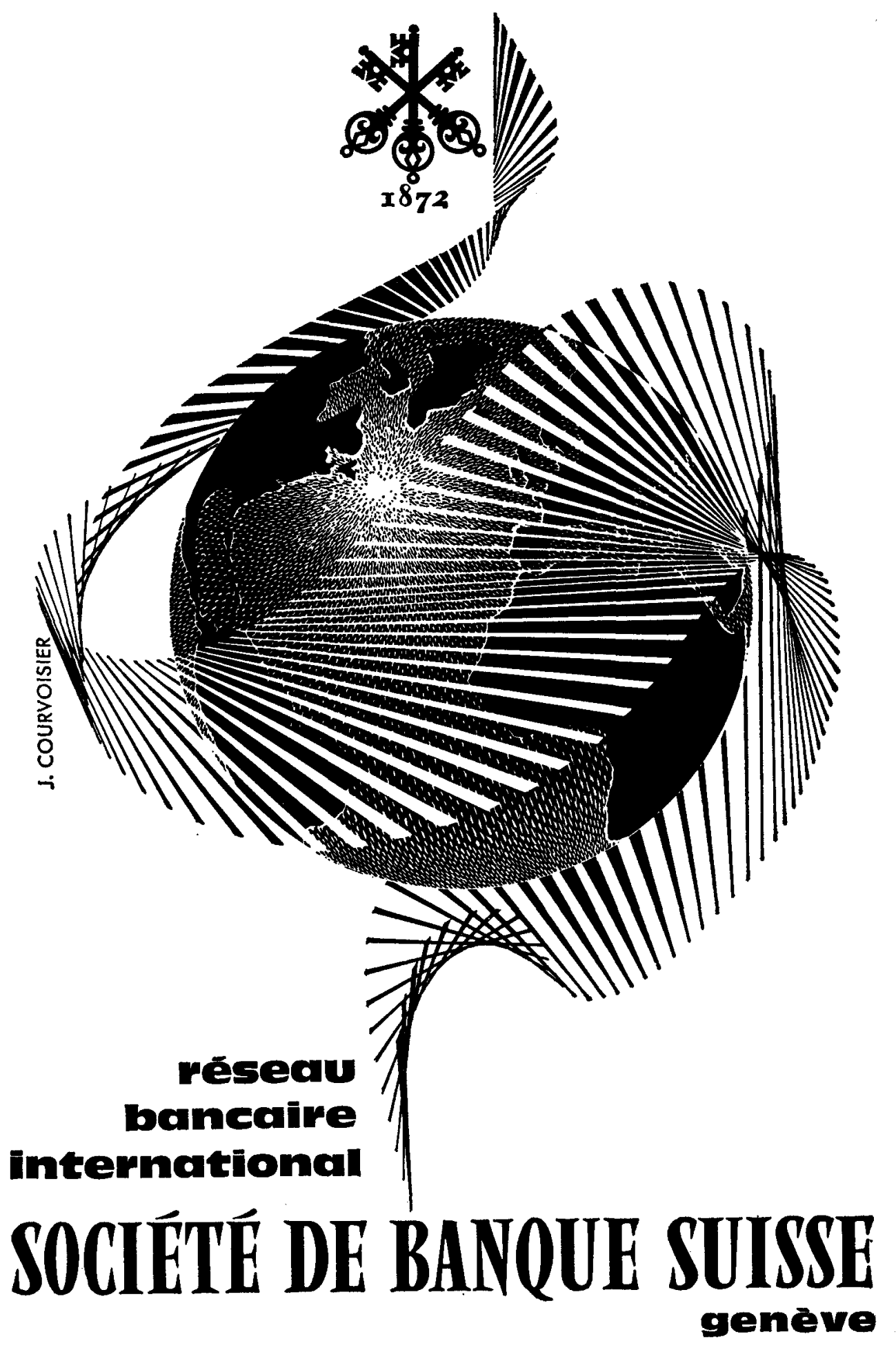




\section{SOCIETE FIDUCIAIRE ROMANDE OFOR S.A.}

Place Saint-Gervais 1, Genève - Tél. 317050

$\downarrow$ Expertises, revisions et organisations comptables

$\downarrow$ Domiciliation et administration de sociétés

$\downarrow$ Assainissements et liquidations

^ Interventions et conseils en matière fiscale

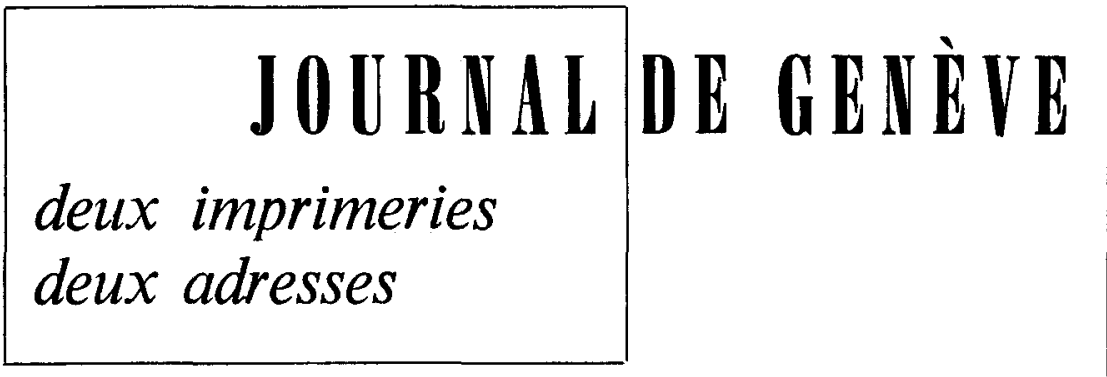

rue François-Dussaud 20

1211 Genève 8 - Téléphone 431940

rue Général-Dufour 5-7

1211 Genève 11 - Téléphone 250350

TYPO - OFFSET - ROTATIVE 


\section{SOMMAIRE}

COMITE INTERNATIONAL

DE LA CROIX-ROUGE

DANS LE MONDE DE LA CROIX-ROUGE

FAITS ET DOCUMENTS

LIVRES ET REVUES

\section{REVUE INTERNATIONALE \\ DE LA CROIX-ROUGE}

DÉCEMBRE 1970 - № 624

V. Segesvary: Comment naquit la solidarité Croix-Rouge - Durant la guerre francoallemande de 1870-1871 . . . . . . . 747

L'action de secours de la Croix-Rouge en Jordanie 773 Activités extérieures:

Le Président du CICR en République fédérale d'Allemagne - En Grèce - Au Proche-Orient - En République du Vietnam - En République khmère - Au Laos - A Hong-Kong Aux Philippines - En Equateur . . . . . 775 *

Activités du Service des secours . . . . . . . . 779

Ligue des Sociétés de la Croix-Rouge: Au secours des victimes du Pakistan oriental . . . . 780 Espagne . . . . . . . . . . . . . . . . . 783

Tunisie. . . . . . . . . . . . . . . . . 784

Journée universelle de l'Enfance . . . . . . 785 Pour la protection de la vie . . . . . . . 786

Table des matières (1970) . . . . . . . 793 
INTERNATIONAL.

REVIEW

OF THE RED CROSS

\section{BUPPLEMENTS \\ De LA ReVue}

\section{EN LANGUE ESPAGNOLE}

EN LANGUE ALLEMANDE

REVUE INTERNATIONALE DE LA CROIX-ROUGE

746
Une édition en langue anglaise paraît chaque mois. Elle est en principe identique à l'édition française, et peut être obtenue aux mêmes conditions.

M. Veuthey: La Cruz Roja y los conflictos sin carácter internacional - Indice, Vol. XXII (1970).

M. Veuthey: Das Rote Kreuz und die nicht-internationalen Konflikte - Inhaltsverzeichnis, Band XXI (1970).

Elle est publiée chaque mois par le Comité international de la Croix-Rouge.

7, avenue de la Paix, 1211 Genève 1 (Suisse)—Compte de chèques postaux $12-1767$.

Abonnement un an: Fr. 25,--; le numéro: Fr, 2,50.

Pour les pays suivants: Algérie, Allemagne, Autriche, Belgique, Danemark, Finlande, France, Italie, Luxembourg, Maroc, Norvège, Pays-Bas, Portugal, Sarre, Suede, Tunisie, Vatican, on peut s'abonner auprès des bureaux de poste.

RÉDACTION: J.-G. LOSSIER

Seuls les textes signés par le Comité international de la CroixRouge engagent la responsabilité de celui-ci. 